\title{
Martensitic transformation plasticity simulations by finite elements
}

\author{
J.F. GANGHOFFER, K. SIMONSSON*, S. DENIS, E. GAUTIER, S. SJÖSTRÖM* and A. SIMON \\ Laboratoire de Science et Génie des Matériaux Métalliques, UA 159 du CNRS, Ecole des Mines, Parc de \\ Saurupt, 54042 Nancy cedex, France \\ * Department of Solid Mechanics and Strength of Materials, University of Linköping, 58183 Linköping, \\ Sweden
}

\begin{abstract}
The mechanical behaviour associated to the martensitic transformation has been modelled using a 2D FE description. The martensite variants are constituted of different elements of the mesh and four different variants are allowed to transform in the grain. The transformation progress is prescribed using a thermodynamical criterion based on the maximal work associated to the variant formation. Transformation plasticity deformation and plates orientation patterns are obtained for three stress levels. These results are discussed in regard to the model used and the physical parameters introduced in the model.
\end{abstract}

\section{INTRODUCTION}

In order to model the transformation plasticity during a martensitic transformation, a micromechanical approach describing the progress of the transformation inside a representative volume element of the material (unit cell) has been established, in order to highlight the role of internal stresses generated on the plates orientations evolution within the cell and on the anisotropic accommodation of transformation strains. Before describing the basic concepts of the model, we first recall briefly some essential physical characteristics of the transformation.

\section{MICROMECHANICAL MODEL OF THE GRAIN}

\subsection{Description of the transformation}

Considering the more specific case of a ferrous alloy, martensitic transformation involves both a shear strain and a volumic variation accompanying the formation of plates within a grain. The plates usually present sharp tips and are limited by the grain boundary or by other already formed plates. A common cristallographic plane between the parent phase and martensite can be observed, called the habit plane. There is consequently a discrete number of its possible orientations, typically 12 for $\{225\} \mathrm{f}$ planes. From a macroscopic point of view, the transformation kinetics is dependent on the temperature and on the stress state $(1,2)$.

According to these main features of the transformation, a model describing the grain behaviour has been established, for a 2-dimensional geometry (fig. 1). The basic cell is a square, meshed with triangular elements in a plane stress situation. The transformation develops by the successive formation of plates within the grain. Each plate consists of a band of elements and we simulate a sharp tip morphology by letting its two end elements untransformed. A habit plane defined by its normal vector $\underline{n}$ and a shear direction $\underline{\mathrm{d}}$ in the plane is associated to each plate. The use of triangular elements allows to describe 4 possible habit plane orientations, namely $0,45,90$ and 135 degrees. 


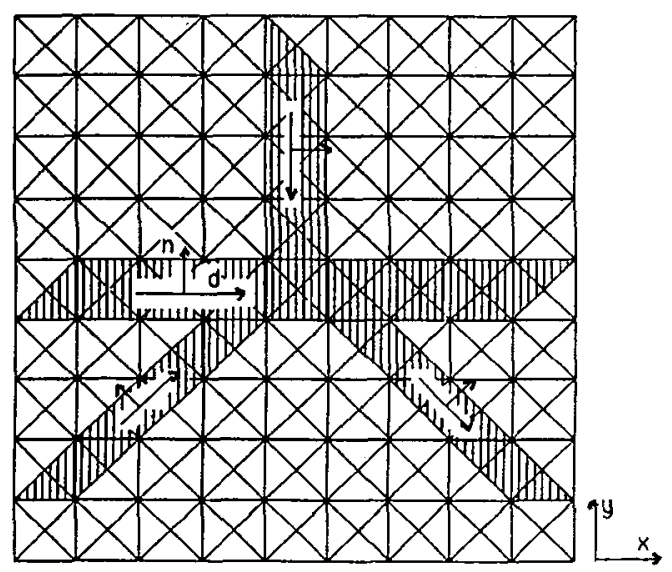

Figure $1:$ Micromechanical model of the grain. FE mesh and associated martensite variants

2.2 Criterion for plates development

In order to establish a criterion for the plates development, a thermodynamical study of a two-phase material (volume $\Omega$ ) separated by a moving interface has been performed [3] based on thermodynamical analysis of the mechanical behaviour of continuum media.The obtained evolution condition takes into account the difference of chemical energy between the phases, the variation of strain energies created on the volume of each phase and the work produced by the transformation progress. It has been shown that the actual transformation process maximizes the work produced and minimizes the increase of strain energy.

As a first approach, we have established a criterion for the development of martensite variants based on the work produced. This work is expressed $\sigma \varepsilon 0+\tau \varepsilon_{0}$ where $\gamma_{0}$ and $\varepsilon_{0}$ characterize the deformation associated to the transformation ( $\gamma_{0}$ is the shear along the habit plane and $\varepsilon_{0}$ the dilatation normal to it) ; $\tau$ and $\sigma$ are respectively the shear stress along the habit plane and the normal stress. The orientation of variants and the order in which they form are then prescribed in the following way : among all untransformed elements within the cell the one for which expression $\sigma \varepsilon_{O}+\tau \gamma_{0}$ reaches its highest value is chosen as a martensite "nucleus" for a plate. Correspondingly, one habit plane orientation is associated to it. It is then assumed that the formation of the plate is instantaneous, its development being stopped either by the grain boundary or by already formed plates.

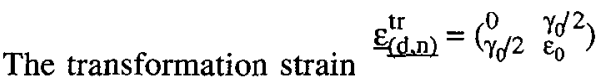
simultaneously to all elements of the plate.

(1) expressed in the local basis $(\underline{\mathrm{d}}, \underline{\mathrm{n}})$ is then imposed

\subsection{Behaviour law of the phases and F.E Simulation}

An elastoplastic behaviour law is used. The input data corresponding to an Fe-Ni-C alloy are listed in Table 1 below. The calculations were performed with the FE code ASKA, using an infinitesimal strain formalism.

Table 1 : Input data for the FE calculation

\begin{tabular}{|l|c|c|c|c|}
\hline \multicolumn{1}{|c|}{ Properties } & $\begin{array}{c}\text { Young's modulus } \\
\text { (MPa) }\end{array}$ & Poisson's ratio & $\begin{array}{c}\text { Yield Strength } \\
(\mathrm{MPa})\end{array}$ & $\begin{array}{c}\text { Hardening slope } \\
\text { (MPa) }\end{array}$ \\
\hline AUSTENITE & $2.10^{5}$ & 0.3 & 300 & 3800 \\
\hline MARTENSITE & $2.10^{5}$ & 0.3 & 1000 & 8300 \\
\hline \multicolumn{4}{|c|}{ Transformation parameters $\gamma_{0}=0.19 ; \varepsilon_{0}=0.03$} \\
\hline
\end{tabular}

Application of suitable boundary conditions on the cell allows to describe the behaviour of a set of interacting grains. Physically, the grain boundary acts as an obstacle to the propagation of plates and we therefore consider an extreme situation of rigid boundaries by prescribing the edges of the cell to keep 
straight and parallel during the transformation progress. The simulation starts by the application of an external load. Application of transformation criterion determines then the next forming plate, which will be given the transformation strain expressed by (1), and new mechanical properties. It follows then an elastoplastic calculation for the plate and the procedure is repeated till the whole mesh is filled with martensite.

\section{RESULTS}

From the FE calculation we get informations about the plate pattern in the grain, the local stress and strain states and the macroscopic deformation of the grain during the transformation. In this paper we have chosen to show only the plate pattern and the variations of the macroscopic deformation with the progress of transformation. The macroscopic total strain is defined as the displacement of one node of the edge perpendicular to the load direction. Moreover we have defined the reversible transformation strain as the accumulated contribution of the transformation strain component in the load direction $\left(\varepsilon_{\mathrm{X}} \mathrm{tr}^{\mathrm{r}}\right)$ for each plate, multiplied by the fraction it represents. Figure 2 shows the components $\varepsilon_{X}{ }^{t r}$ for the different possible habit plane orientations. This definition of a macroscopic transformation strain provides an evaluation of the stress-induced anisotropy of the deformation associated with the plate orientation distribution. The difference between the macroscopic total strain and the transformation strain defines then the macroscopic resulting elastic and plastic strain. We analyse here the results for different applied stresses 10,100 and 250 MPa in uniaxial tension (direction $\mathrm{x}$ ). These stresses are below the yield stress of austenite.

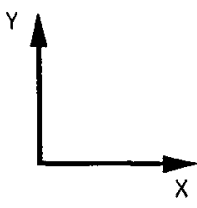

$\gamma=\mathbf{0 , 0 1 5}$

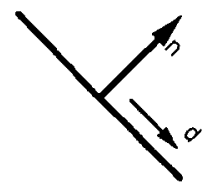

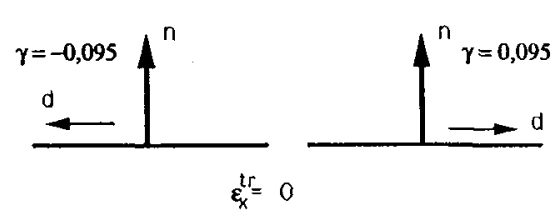

$\gamma=-0,015$
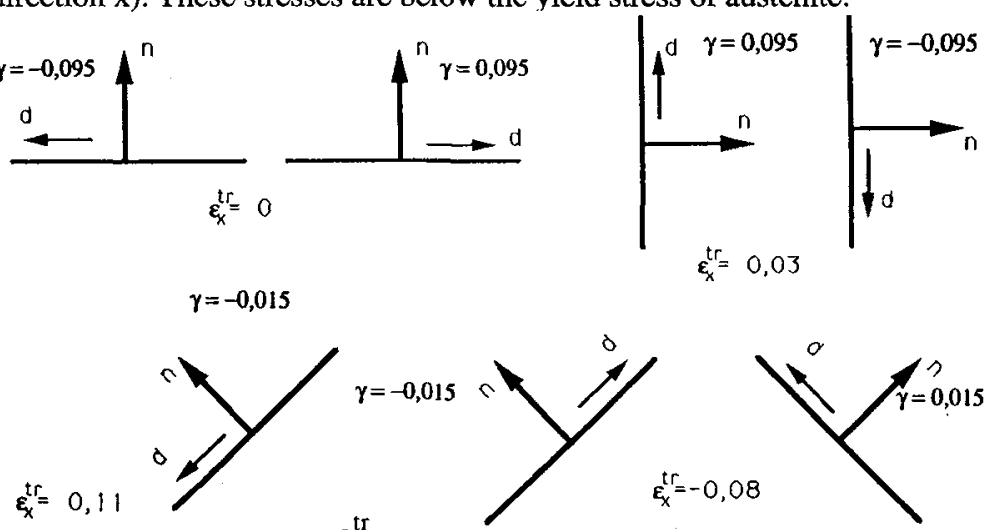

$\mathbf{E}_{x}^{t}=-0,08$

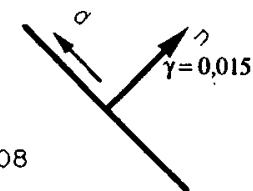

Figure 2: Deformations associated to the variants: $\varepsilon_{\mathrm{x}}^{\mathrm{tr}}$ contribution of the transformation strain in the $\mathrm{x}$ direction $\gamma$ transformation shear contribution.

The application of a small load (10 MPa) leads to the plate pattern shown on figure 3a. The first plate represents a transformed fraction of about $3 \%$; the immediately next forming plates $(2,4)$ receive other orientations and give rise to plastic strains (fig. 3b). The resulting pattern is such that no marked anisotropy is observed, some bands keeping the orientation of the first one (e.g. bands 6,10,17,29,35), alternating with horizontal and vertical bands (e.g. bands $2,4,11,16,25,28,37$ ). The main contribution of such vertical bands occurs between about $50 \%$ and $70 \%$ transformed fractions and a small positive plastic strain is measured during this interval (both opposite shear directions lead to a positive contribution of the strain in the load direction).As a consequence of the plate pattern, there is a quasi linear evolution of the strains ; particularly, the evolution of the total strain is nearly the one that would be obtained for an equivalent contribution of the variants, leading to a final value equal to half the volumic variation, i.e. $1.5 \%$ (total strain values in respective directions are $\mathrm{E}_{\mathrm{X}}=1.4 \% ; \mathrm{E}_{\mathrm{y}}=1.2 \%$ ). One concludes from these results that an external stress of $10 \mathrm{MPa}$ has a small influence on the variants orientations and leads to a near isotropic behaviour of the cell. Results of the calculation with a $250 \mathrm{MPa}$ external load are presented on fig. 4a and 4b. The macroscopic strain is extremely large in that case (up to $13 \%$ ), due to the cumulative contributions of favourably oriented plates. A difference with the run using $10 \mathrm{MPa}$ occurs from the very beginning since the second plate is oblique for the higher load (it is vertical for $10 \mathrm{MPa}$ ). Very few horizontal and vertical bands form in that case and most of the oblique bands (with either $45^{\circ}$ or $135^{\circ}$ orientation of their habit plane) present the same shear direction. Applying a stress state near the austenite yield strength leads to 

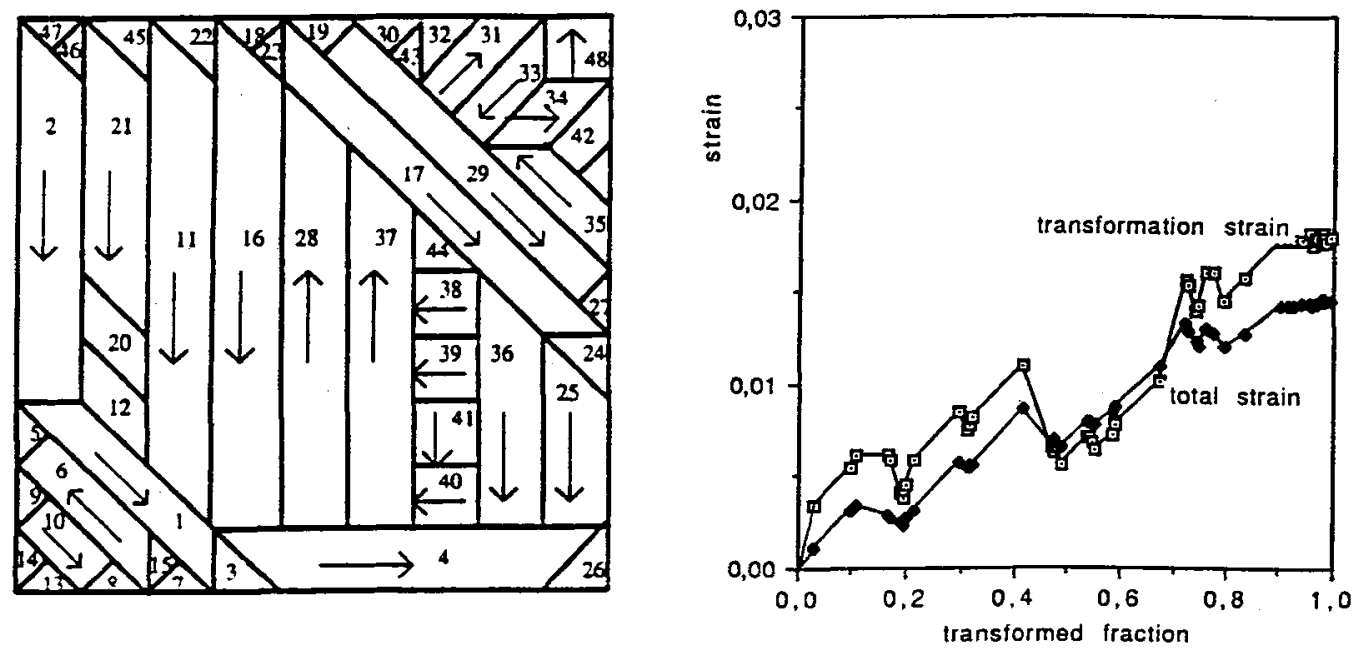

Figure 3 : Plate pattern and deformations evolution (in the $\mathrm{x}$ direction) for a transformation under $10 \mathrm{MPa}$ internal stress levels of the same order of magnitude as the external load. The effect of internal stresses on the plate development is therefore much smaller as compared to the case with $10 \mathrm{MPa}$. Consequently applying $250 \mathrm{MPa}$ leads to a marked preferential orientation of the plates all along the transformation : the external stress plays the major role in the plate orientation determination. This is further evidenced by the quite linear evolution of the transformation strain (fig. 4b) in that case and by the occurence of large and more evenly distributed plastic flow from the beginning. Anisotropic plastic accommodation of transformation strains represents a large contribution to the total strain (about $50 \%$ ).
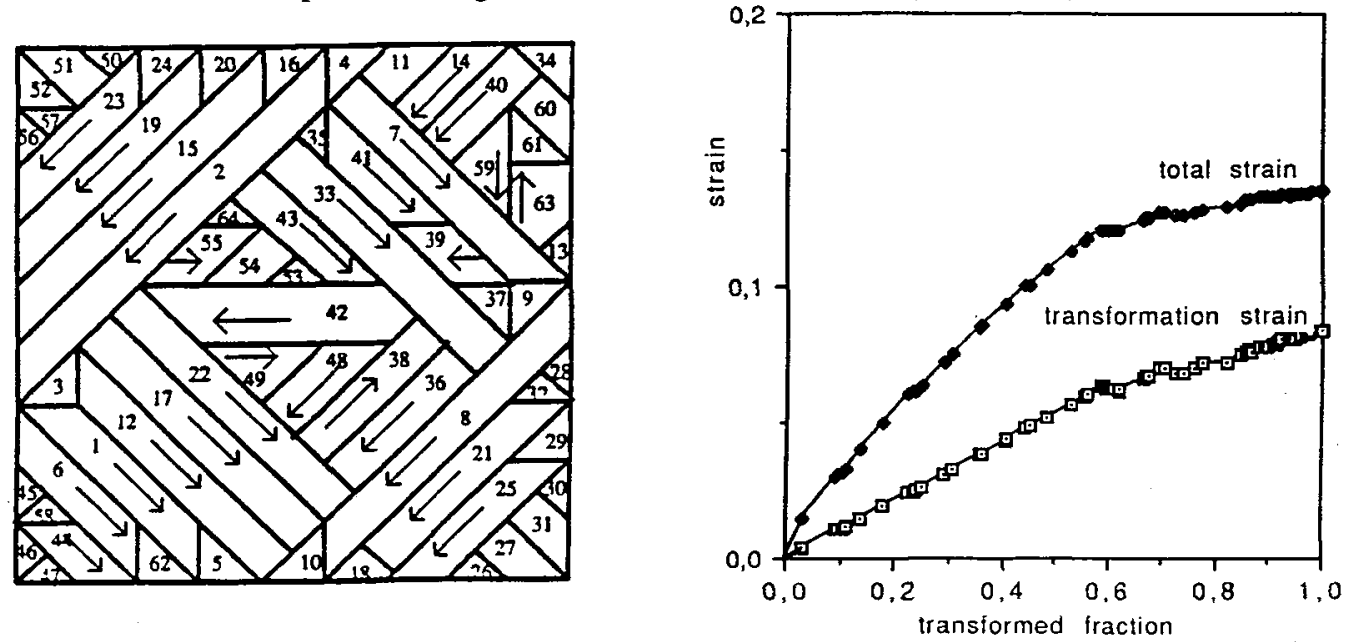

Figure 4 : Plate pattern and deformation evolutions for a transformation under $250 \mathrm{MPa}$.

Application of a $100 \mathrm{MPa}$ external stress (the calculation has been performed till about $60 \%$ transformed fraction) shows deformation levels (fig. 5) about three times smaller compared to those obtained for 250 $\mathrm{MPa}$. Nevertheless, the evolutions of transformation strain are nearly the same for the two stress levels till a fraction of about $20 \%$, more plastic flow being generated for 250 than for $100 \mathrm{MPa}$. These comparisons show that the local plastic flow doesn't influence very much the variants orientations distribution in the model used.

In order to make a first comparison of the simulation with experience, we have reported on fig. 6 the evolutions of the total strain versus transformed fraction in both cases, for a $250 \mathrm{MPa}$ applied load. Experimental results are obtained for an $\mathrm{FeNiC}$ alloy [1]. The shape of the deformation evolutions is similar, showing a high slope at the beginning due to a favorable orientation effect of the variants, which 
decreases with the progress of the transformation. However, the simulation overestimates considerably the deformation levels (final values are 4 times higher).

Moreover, microstructural observations did not show such large orientation effects (the model gives for $50 \%$ transformation the most favorable orientation for all the plates).

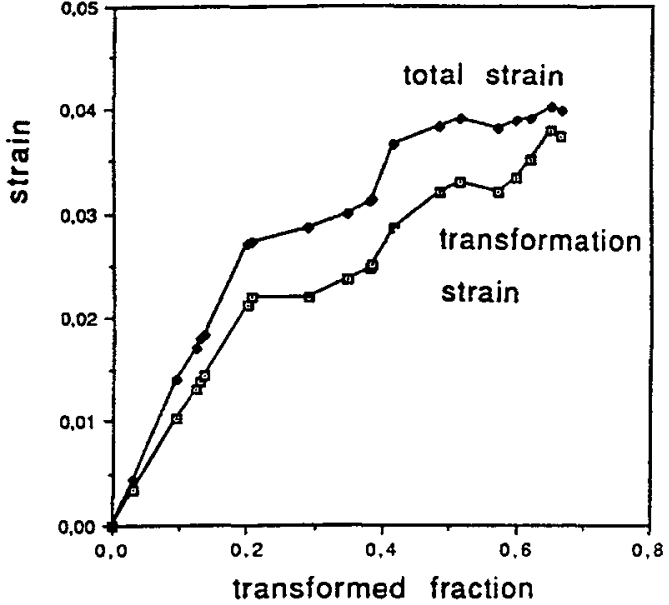

Figure 5 : Deformation evolutions for a transformation measured under $100 \mathrm{MPa}$.

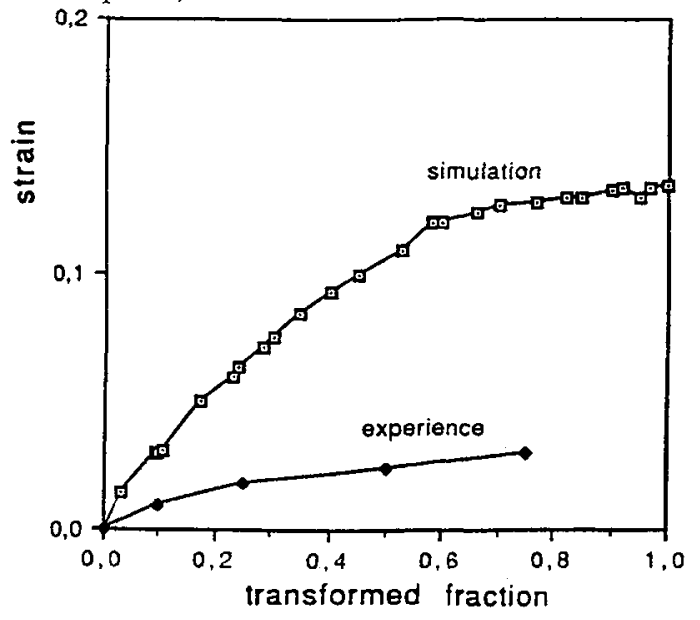

Figure 6 : Calculated deformation and measured transformation plasticity deformation for $250 \mathrm{MPa}$.

\section{DISCUSSION}

Our results show that if the present model allows to describe the general shape of the evolution of transformation plasticity, it is not sufficient to give a quantitative prediction. The two main points which may affect the calculated results that we discuss in the following are : the effect of the parameters inherent to the FE model and the physical parameters.

\subsection{Effect of the FE model parameters}

Since the morphology of the variant is closely related to the FE discretization in this $2 \mathrm{D}$ model, the size of the mesh determines both the transformed fraction at each step, the aspect ratio of the variants (their width divided by their length) and the resulting evolutions of the strains at the cell level. For the mesh size used in the calculations (fig. 1), the smallest aspect ratio is about 0.07 . The formation of such variants leads to a plastic flow which is localized at their tip and the stress state along their edges is near the yield strength of austenite. The progress of the transformation leads to the building of shorter plates, which induce higher plastic flow along the variant. Experimentally, one generally observes thin plates. A finer mesh would allow to get closer to the actual morphology of plates, which could modify their distribution and the resulting macroscopic strains. The nature of boundary conditions on the cell is essential since it describes the interactions between neighbouring grains, which in turn interacts with the development of the variants within the cell. The prescribed rectangularity of the cell corresponds to an extreme case of strong interactions between grains - the planes of the cell are considered in that case as symmetry planes preventing any distorsion of the boundary. Such a situation leads to very high plastic strain levels when a variant reaches the grain boundary and it seems therefore more realistic to allow a smother description of the interactions between grains.Moreover, we use a $2 \mathrm{D}$ model with plane stress conditions. A similar model with plane strain conditions may give another bound of the mechanical behaviour. Nevertheless, to describe the real situation, a 3D model would be necessary.

\subsection{Physical parameters}

Different parameters must be considered when we compare the experimental and calculated results : the different lattice orientations of the grains, the mechanical properties of the phases and the transformation 
description. Considering the first one, experimental results are obtained for a macroscopic specimen, which implies an averaging effect, particularly over the lattice orientations of the grains. Considering this parameter in the simulation could lead to a more isotropic behaviour of the cell. For the second one, the transformation involves high interfacial velocities, which in turn implies high deformation rates within the bulk phase. They can lead to higher mechanical properties for austenite when it is transformed in martensite, as compared to the one we have considered in the simulation, which are obtained for stable phases. In order to estimate the effect of mechanical properties of the phases on the behaviour of the material, the extreme case of elastic phases has been considered and a calculation for a $250 \mathrm{MPa}$ applied stress has been performed. We obtain a linear variation of the transformation strain (which is nearly similar to the total strain because elastic strains are very low) with the increase of martensite all over the transformation due to a maximal orientation effect. For a complete transformation, the total strain of 0.11 is less high compared to that obtained for the previous elastoplastic calculation (0.13). However in the elastoplastic case, half of the total contribution is due to the preferential orientations of martensite plates and half is due to anisotropic plastic accommodation. Also, using an elastic behaviour reduces this last part, but increases the preferential orientation contribution.

At least, one essential feature of the model is the description of the transformation progress, which relies on a thermodynamical criterion considering the maximum work produced, associated to an instantaneously formation of the plate. The effect of strain energies of the phases that has been neglected in this approach can be important, considering the growth of the plate. It can lead to stop its progression and the choice of an other habit plane, in order to minimize the increase of strain energy over the cell. More generally, the formulation of an evolution criterion plays an important role in the way mutual interactions between variants are described. The formation of plates in the present model is accommodated by a plastic flow localized at the plate ends. However, the formation of self-accommodating plates which is observed experimentally is not described in the model. Such self-accommodating plates tend to minimize the transformation strain and the strain energy generated. It is therefore necessary to make further work in order to get a criterion that leads to a better representation of accommodation by transformation.

\section{CONCLUSION}

The different concepts necessary for a micromechanical description of a martensitic transformation in a grain have been introduced in a FE calculation. The model allows to describe the influence of the internal stress state on the selection of variants orientations and progress of the transformation. The global deformation of the grain can be separated in two contributions which quantify the two mechanisms behind transformation plasticity: the part of the deformation due to the orientation of the martensite plates and the deformation due to plastic accommodation. Results have been obtained for different uniaxial applied stress states and a first comparison with experience shows that the form of the evolutions of the total strain is similar. However, the simulation overestimates the strain levels, and the model shall be extended further in order to handle the more realistic case of a 3-D situation, as to integrate a criterion leading to a minimization of mechanical accommodation energies.

\section{AKNOWLEDGMENTS}

The work described has been supported jointly by CNRS (the French Center of Scientific Research) and STU (the Swedish Board for Technical Development). The computer calculations have been performed on a CRAY XMP/48 at NSC (the National Supercomputer Center, LINKÖPING, Sweden).

\section{REFERENCES}

/1/ GAUTIER E., SIMON A., COLLETTE G. and BECK G. ; J. de Physique Colloque C4, $\sup n^{\circ} 1243,(1982) 473$.

GAUTIER E., SIMON A. and BECK G. ; Proc. International Conference on Martensitic

Transformation, (J.I.M) (1986) 502.

12/ DENIS S., GAUTIER E., SIMON A. and BECK G. ; Mat. Sci. Techn. 1 (1985) 805.

13) GANGHOFFER J.F., DENIS S., GAUTIER E., SIMON A., SIMONSSON K. and SJÖSTRÖM S. ; J. de Physique IV, sup. n 11 Colloque C4 vol 1 (1991) 89.

141 CHRISTIAN J.W.; The theory of transformations in Metals and Alloys (Pergamon Press, Oxford), 1975. 